F. D. Kahn

Department of Astronomy

University of Manchester

\title{
1. THE EXPLOSION
}

Supernovae occur in highly evolved, massive stars and typically release $10^{51}$ to $10^{52} \mathrm{erg}$. Their light curves show that the stellar radius is about one $A . U$. at the time of the explosion. The typical energy density in the star is about $10^{11}$ or $10^{12} \mathrm{erg} \mathrm{cm}^{-3}$ when the blast reaches the stellar surface. Much of the energy is present in thermal form. Since the density of the material is high, thermal equilibrium is attained rapidly between the radiation field and the thermal motions. The temperature in the interior of the star becomes about $10^{6} \mathrm{~K}$, and most of the energy is present in the form of radiation.

At this time the star has a large optical depth, of order $10^{7}$. Therefore the effective diffusion speed for radiation is very low, about $3000 \mathrm{~cm} \mathrm{~s}^{-1}$. But the effective sound speed is large, of order $10^{9} \mathrm{~cm} \mathrm{~s}^{-1}$, and the large input of energy has destroyed the gravitational equilibrium. So the star begins violently to expand, and the pressure of the stored radiation field does work in producing mass motion of the material. Only a small fraction of the radiant energy is released from the exploded star. The photosphere, in this phase, lies in the region where the gas changes from being ionized to non-ionized, so that the photospheric temperature remains low, of order $10^{4} \mathrm{~K}$. There is 1 ittle chance of observing any high-temperature phenomena at this stage in the evolution of a supernova.

In the end, the remnant become a mass of cool gas with a high expansion speed, of order $10^{4} \mathrm{~km} \mathrm{~s}^{-1}$.

\section{INTERACTION WITH THE INTERSTELLAR MEDIUM}

Interaction with the interstellar medium becomes interesting after a hundred years or so. Conventionally, there are three phases:

Phase I, during which the mass of swept-up interstellar gas is less than the mass ejected from the exploded star, 
Phase II, during which the mass of swept-up interstellar gas exceeds the mass ejected from the exploded star, but there has not yet been time enough for appreciable energy-loss by radiative cooling, and

Phase III, after radiative cooling has become important for the dynamics of the remnant.

\subsection{The Early Stages}

During Phase I a very strong shock sweeps forward into the interstellar gas. The whole remnant is now optically thin, and the radiation field is far from thermal equilibrium with the gas kinetic motions in the gas. Therefore the shocked interstellar gas becomes intensely hot, with a temperature of order $10^{9} \mathrm{~K}$.

A second shock moves inward relative to the gas ejected from the central star. It is initially rather weak, since the deceleration is only small to begin with, but eventually strengthens. The shocked gas from the star starts off by being much cooler than the shocked interstellar gas, but towards the end of Phase I, which lasts a few hundred years, cooling times are long and radiative losses are dynamically quite unimportant.

This description is rather qualitative and highly idealised. The radio source Cassiopaeia $A$ is the usual example given for a supernova in Phase I. It is indeed observed to contain gas at different densities and temperatures, but its overall appearance and velocity pattern are much less simple than the present idealised picture suggests.

\subsection{The Standard Treatment for Phase II}

Traditionally Phase II is described in terms of the Sedov solution, which applies if

(i) the swept-up mass far exceeds the ejected mass

(ii) there has been little radiative cooling

(iii) the ambient interstellar gas has little energy density

(iv) and is uniformly distributed before the blast wave strikes it and (v) transport phenomena (viscosity and heat-conduction) are everywhere negligible, except in the shock.

It is by no means clear that these conditions are ever completely satisfied. Much recent work has treated the effects of supernovae on a lumpy interstellar medium, and the consequences of thermal conduction by electrons in redistributing the heat within a remnant. The concept of the lumpy medium comes from the many observations which show that the bulk of the interstellar mass normally resides in small, cool clouds of high density, and that much interstellar space is filled by a tenuous but hot $\left(10^{6} \mathrm{~K}\right)$ gas. Any blast wave in such a medium would deposit most of its energy in the low density component, and produce a SNR with an appearance radically different from that which is observed. 
Again, even if the ambient medium is more or less uniform, there will be significant differences depending on presence or absence of large-scale heat conduction. Observation appears to favour the description in terms of an adiabatic medium.

\subsection{Predictions of the Sedov Solution}

Now during Phase II the total energy of a remnant is conserved, but there are continued adjustments in the energy content of the different positions of gas within it. The state of motion is defined by two parameters, Eothe energy and $\rho_{0}$ the ambient interstellar density. At time $t$, the shock radius is

$$
r_{S}=a t^{2 / 5}
$$

where

$$
a^{5}=2 E_{0} / \rho_{0}
$$

The post-shock pressure is

$$
P_{s}=\frac{3}{25} \quad \rho_{0} a^{2} t^{-6 / 5}
$$

and the post-shock density

$$
\rho_{S}=4 \rho_{0}
$$

The mass included within radius $r_{s}$ is

$$
\begin{aligned}
& M_{S}=\frac{4 \pi}{3} \rho_{0} a^{2} t^{6 / 5} \\
& \kappa=P / \rho^{5 / 3}
\end{aligned}
$$

then the specific entropy is

$$
\mathrm{S}=\frac{3 \mathrm{k}}{2 \mathrm{~m}} \log \mathrm{K}
$$

and $S($ or $k$ ) is conserved, following the motion of the fluid, if transport processes are not important, and radiative cooling is negligible. Immediately behind the shock,

$$
K=\frac{2^{2 / 3} \pi \rho_{0} a^{5}}{100 M}
$$

and this relation remains valid for any portion of the fluid until conduction and/or radiative cooling become significant.

In Phase II, most of the mass of the shocked gas lies in a relatively thin shell, with outer radius $r_{s}$; in the interior of the remnant, say for $r$ less than $0.9 \mathrm{r}_{s}$, the pressure is almost uniform and equal to $0.31 \mathrm{P}_{\mathrm{s}}$. In the inner region of the remnant the run of density and temperature are, respectively, given by 


$$
\rho \doteqdot 0.35 \rho_{s}\left(r / r_{s}\right)^{9 / 2}
$$

and

$$
\mathrm{T} \doteqdot 0.88 \mathrm{~T}_{\mathrm{S}}\left(\mathrm{r}_{\mathrm{S}} / \mathrm{r}\right)^{9 / 2}
$$

The mass included within radius $r$ is

and

$$
M \div 0.57 \mathrm{M}_{\mathrm{s}}\left(\mathrm{r} / \mathrm{r}_{\mathrm{s}}\right)^{15 / 2}
$$

$$
\kappa \doteqdot 1.75 K_{s}\left(r_{s} / r\right)^{15 / 2}
$$

The thermal energy within radius $r$ is

$$
\mathrm{E} \doteqdot 0.47 \mathrm{E}_{0}\left(\mathrm{r} / \mathrm{r}_{\mathrm{s}}\right)^{3}
$$

Even during the Sedov phase there will be radiative cooling, but at first it has no significant effect. It is usually described in terms of a cooling function $\Lambda(T)$; in the temperature range $10^{5} \mathrm{~K}<\mathrm{T}<3 \times 10^{7} \mathrm{~K}$, a good approximation is

$$
\Lambda(\mathrm{T}) \doteqdot 1.3 \times 10^{-19} \mathrm{~T}^{-1 / 2}
$$

so that the rate of change, following the motion of the fluid, is given by

$$
\frac{D}{D t} k^{3 / 2}=-q=-4 \times 10^{32}
$$

after substitution of typical values. The Sedov phase will certainly have ended when the newly shocked gas has a cooling time equal to the age of the remnant. The cooling time is the period needed to reduce $k$ to zero. Typically, the Sedov phase ends when the shock has slowed to about $250 \mathrm{~km} \mathrm{~s}^{-1}$ and the remnant is some 30000 to 50000 years old.

During Phase II, the mass motion contributes about a quarter of the remnant's energy and the rest is thermal. When the shock speed has fallen to about $250 \mathrm{~km} \mathrm{~s}^{-1}$ the material speed in the shell will be three quarters as large, say $200 \mathrm{~km} \mathrm{~s}^{-1}$ and the energy due to mass motion some $2 \times 10^{14}$ erg $\mathrm{gm}^{-1}$. Thus the average energy per unit mass at this stage is $8 \times 10^{14} \mathrm{erg} \mathrm{gm}^{-1}$, compared with some $10^{18} \mathrm{erg} \mathrm{gm}^{-1}$ in the mass originally ejected from the exploded star. No significant amount of energy has yet been lost from the remnant. Consequently, the swept-up mass at the end of Phase II is about a thousand times larger than the ejected mass. 


\section{PRODUCTION OF HOT GAS AND ITS CONSEQUENCES}

Now the predictions of the Sedov model are good only for the sweptup mass. It therefore follows from relation (11) that they apply only to the material with radial distance larger than $0.43 r_{s}$, even at the end of Phase II. So, for example, it seems permissible to estimate the cooling time for the gas at $r=\frac{1}{2} r_{s}$. By relation (12) the value of $k^{3 / 2}$ is there some $5640 \times \mathrm{K}_{\mathrm{S}}^{3 / 2}$. Clearly this gas has a correspondingly longer cooling time than the gas immediately behind the shock, and would stay hot, according to the present assumptions, for some 200 million years. But the amount of gas involved is sma11, some $0.3 \%$ of the mass of the remnant, and Iong before this time has passed the gas would have escaped into the galactic halo. It is probably more relevant to calculate what fraction of the mass can stay hot for $10^{6}$ years, for this is about the time needed for the gas to drift to the edge of the galactic disk. Here the minimum value of $K$ is given by

$$
\mathrm{K} \gg 25 \mathrm{~K}_{\mathrm{s}}^{3 / 2}
$$

so that

$$
\mathrm{k} \geqslant 8.5 \mathrm{~K}_{\mathrm{s}}
$$

and the mass which stays hot is given by

$$
\mathrm{M} \div 0.12 \mathrm{M}_{\mathrm{S}}
$$

Possibly this supply of gas will be adequate for the halo. A generous requirement is that a wind with a density of $10^{-2}$ atoms $\mathrm{cm}^{-1}$ should leave the disk with a speed of $10^{7} \mathrm{~cm} \mathrm{~s}^{-1}$. This estimate can easily be related to models of supernova remnants.

At the end of Phase II the average energy in the remnant will be $8 \times 10^{14} \mathrm{erg} \mathrm{gm}^{-1}$. If the energy of the supernova was $E_{0}$, the corresponding mass of the remnant is $1.25 \times 10^{-15} \mathrm{E}_{0}$ and about $12 \%$ of this becomes available for the wind into the halo. For an adequate supply of hot gas, supernova explosions in the Galaxy must release about $6 \times 10^{42} \mathrm{erg} \mathrm{s}$, on average. If a typical explosion has an energy of $10^{52} \mathrm{erg}$, the required rate of such events is one every fifty years in the Galaxy - or correspondingly more if the typical supernova energy is less. The gas that cannot cool in $10^{6}$ years has

$$
K \geqslant 5 \times 10^{30}
$$

and, when it has expanded to an interstellar pressure of $10^{-12}$ dyne cm $\mathrm{cm}^{-1}$, will have a mean density $1.5 \times 10^{-26} \mathrm{gm} \mathrm{cm}^{-1}$, and a mean temperature of about $5 \times 10^{5} \mathrm{~K}$.

Finally, at the end of Phase II, the immediate post-shock temperature will be $7 \times 10^{5} \mathrm{~K}$. At interior points the gas is much hotter, with a temperature of $1.7 \times 10^{6} \mathrm{~K}$ at $\mathrm{r}=0.8 \mathrm{r}_{\mathrm{s}}$, and $1.4 \times 10^{7} \mathrm{~K}$ at $\mathrm{r}=0.5 \mathrm{r}_{\mathrm{s}}$. 


\section{LIMITS ON THE EFFECTS OF THERMAL CONDUCTION}

There are well-known formulae for the electron thermal conductivity in a plasma. They are based on calculations in which the charged particles interact via binary encounters. For the hot and tenuous gas within the SNR these formulae can be used to predict that heat conduction should be highly effective until the end of the Sedov phase. If so, it would remove the differences in temperature which are an essential feature of the Sedov model. But plasma effects set a limit to the rate at which the electrons can carry heat through the plasma.

In the first place, one expects ion acoustic instabilities if the peak in the electron thermal velocity distribution is displaced from the peak in the ion velocity distribution by more than the ion thermal speed. But this effect is important only for electrons with small velocity components in the direction of the temperature gradient, and they do not carry much energy. It seems more relevant to $100 \mathrm{k}$ for other plasma effects.

Thermal conduction can only occur along the magnetic lines of force. Even in a gas at a temperature of $10^{7} \mathrm{~K}$ the electrons have a Larmor radius $r_{L}$ equal to $60 / \mathrm{H} \mathrm{cm}, \mathrm{H}$ being measured in gauss. For any imaginable magnetic field, $r_{L}$ will be minute in comparison with the dimensions of a supernova remnant, and the electron motions will be guided by the lines of force. But the interstellar magnetic energy density will always be so small that the magnetic field has negligible effect on the overall dynamics of a remnant, even at the end of Phase II.

Suppose that prior to the explosion the interstellar magnetic field was uniform, with field strength $H_{0}$. To calculate its later distortion, consider the gas that was initially in a sphere of radius $r_{0}$. Later, when the remnant has reached a radius $r_{s}$, let this sphere have a radius $r$, so that

$$
\begin{aligned}
M=\frac{4 \pi}{3} \rho_{0} r_{0}{ }^{3} & =0.57 M_{s}\left(r / r_{s}\right)^{15 / 2}=0.57 \times \frac{4 \pi}{3} \rho_{0} r_{s}^{3}\left(r / r_{s}\right)^{15 / 2} \\
r & =1.12 r_{s}^{3 / 5} r_{0}^{2 / 5}
\end{aligned}
$$

Let the polar angle $\theta$ be defined with respect to the initial magnetic field direction. Then a line of force which originally passed through $\left(r_{0}, \theta\right)$ will later pass through $(r, \theta)$, with $r$ given by equation (21). Initially,

$$
p_{0} \equiv r_{0} \sin \theta
$$

is the same for all points on a given line of force. Later the line is distorted to a shape given by

$$
\mathrm{r}=1.12 \mathrm{r}_{\mathrm{s}}^{3 / 5} \mathrm{p}_{0}^{2 / 5} \operatorname{cosec}^{2 / 5} \theta
$$

in the interior of the remnant. Take a line of force which meets the 
outer shock at distance $p_{0}$ from the axis of symmetry. It will cut the equatorial plane at distance $p$ from the centre, such that

$$
\mathrm{p} / \mathrm{r}_{\mathrm{s}}=1.12\left(\mathrm{p}_{0} / \mathrm{r}_{\mathrm{s}}\right)^{2 / 5}
$$

The lines of force from the very inner region, say $r<0.5 r_{s}$, therefore run through a much smaller polar cap on the supernova shell, with $\mathrm{p}_{0}<0.13 \mathrm{r}_{\mathrm{s}}$. For the interior region with $\mathrm{r}<0.8 \mathrm{r}_{\mathrm{s}}$, the lines run to a cap with $\mathrm{p}_{0}<0.43 \mathrm{r}_{\mathrm{S}}$. Any heat from the inner part of the remnant is conducted preferentially to the polar regions of the remnant, and this will tend to introduce strong departures from spherical symmetry.

But if there is heat conduction, then on a microscopic scale the electron thermal velocities cannot be isotropically distributed in the rest frame of the hot plasma. To illustrate the consequences, consider a plasma in which there is just one electron speed for any direction, so that

$$
v=v_{0}(1+\varepsilon \mu)
$$

where $\cos ^{-1} \mu$ is the angle between the direction concerned and the line of force. In the plasma rest frame there can be no overall flow of electrons, so that the electron number density also depends on $\mu$, and to first order,

$$
f=\frac{n_{0}}{4 \pi}(1-\varepsilon \mu)
$$

is the number of electrons per unit volume and unit solid angle in direction. The pressure will be anisotropic; in the usual notation,

$$
P_{u}=\frac{1}{2} n_{0} m v_{0}^{2} \int_{-1}^{1}(1-\varepsilon \mu)(1+\varepsilon \mu)^{2} \mu^{2} d \mu
$$

and

$$
P_{\perp}=\frac{1}{2} n_{0} m v_{0}^{2} \int_{-1}^{1}(1-\varepsilon \mu)(1+\varepsilon \mu)^{2}\left(\frac{1-\mu^{\prime}}{2}\right) \mathrm{d} \mu
$$

To second order in $\varepsilon$

$$
\begin{aligned}
\Delta \mathrm{P} \equiv \mathrm{P}_{\perp}-\mathrm{P}_{\|} & =-1 / 4 \mathrm{n}_{0} \mathrm{~m} \mathrm{v}_{0}^{2} \int_{-1}^{1} \varepsilon^{2} \mu^{2}\left(1-3 \mu^{2}\right) \mathrm{d} \mu \\
& =2 / 5 \mathrm{P} \varepsilon^{2}
\end{aligned}
$$

with

$$
\mathrm{P} \equiv 1 / 3 \mathrm{n}_{0} \mathrm{~m} \mathrm{v}_{0}^{2}
$$

The rate of transport of thermal energy by the electrons is

$$
\begin{aligned}
\Phi & =1 / 4 \mathrm{n}_{0} \mathrm{~m} v_{0}^{3} \int_{1}^{1}(1-\varepsilon \mu)(1 \varepsilon \mu)^{3} \mu d \mu \\
& =1 / 3 \mathrm{n}_{0} \mathrm{~m} \mathrm{v}_{0}^{3} \equiv P v_{0} \varepsilon
\end{aligned}
$$


Counting the ion contribution, the thermal energy density in the plasma is $3 \mathrm{P}$, so that the effective speed of heat transport $v_{\operatorname{diff}}$ is $1 / 3 \varepsilon v_{0}$. But there will be a mirror instability unless

$$
\Delta \mathrm{P}<\mathrm{H}^{2} / 8 \pi
$$

Violation of this inequality will halt the heat flow. Thus, setting $v_{m, e}$ equal to the typical electron thermal speed,

$$
v_{\text {diff }} \leqslant \frac{1}{3}\left(\frac{5 \mathrm{H}^{2}}{16 \pi \mathrm{P}}\right)^{\frac{1}{2}} \mathrm{v}_{\mathrm{m}, \mathrm{e}}
$$

When values are substituted from the Sedov model, the right hand side increases with radial distance $r$.

For a rough estimate of the upper limit, set $\mathrm{H}=\mathrm{H}_{0}$ and

$$
\mathrm{P}=0.31 \mathrm{P}_{\mathrm{S}}=0.23 \rho_{\mathrm{o}} \mathrm{v}_{\mathrm{S}}^{2}
$$

and obtain $v_{\text {diff }} \leqslant 0.78 v_{A} v_{m, e} / v_{s}$

where $v_{A}$ is the Alfven speed in the interstellar gas ahead of the shock. If appreciable heat transport is to occur, $v_{d i f f}$ must at least be comparable with $\mathrm{v}_{\mathrm{S}}$ and so

$$
v_{s}<0.88\left(v_{A} v_{m, e}\right)^{\frac{1}{2}}
$$

Now defining $v_{\mathrm{m}} \equiv\left(\mathrm{kT} / \overline{\mathrm{m}}^{\frac{1}{2}}\right.$, then allowing for ions and electrons, $\overline{\mathrm{m}}=\mathrm{m}_{\mathbf{i}} / 2$ and

$$
v_{m, e}=\left(m_{i} / 2 m_{e}\right)^{\frac{1}{2}} v_{m}
$$

In the outer part of the remnant

$$
\mathrm{v}_{\mathrm{m}}=(3 / 16)^{\frac{1}{2}} \mathrm{v}_{\mathrm{s}}
$$

so that there will be appreciable heat flow only if

$$
v_{s}<0.24\left(m_{i} / m_{e}\right)^{\frac{1}{2}} v_{A}=12 v_{A}
$$

The interstellar Alfven speed, for a density $\rho_{0}=10^{-24} \mathrm{gm} \mathrm{cm}^{-3}$ and a field $\mathrm{H}_{0}=3 \times 10^{-6}$ gauss, will be

$$
\mathrm{v}_{\mathrm{A}}=8 \mathrm{~km} \mathrm{~s}^{-1}
$$

Heat conduction cannot become important unless the shock speed has dropped below $100 \mathrm{~km} \mathrm{~s}^{-1}$. But that occurs long after the end of Phase II. 


\section{THE INFLUENCE OF INHOMOGENEITIES IN THE INTERSTELLAR MEDIUM}

There remains the problem of the irregular structure of the interstellar medium. If a supernova explodes into an average region of the interstellar medium, then the resulting blast wave will move out very fast into the inter-cloud gas and much more slowly into the clouds. This will result in a remnant with a structure that differs markedly from those observed or from that predicted by the Sedov solution.

But the medium around the site of a supernova is in general not typical of interstellar space as a whole. Supernovae develop from bright stars, and bright stars tend to ionize and evaporate dense clouds. Therefore, in the region where a supernova explodes, the gas should be rather more diffuse than is typical in interstellar space, and so the Sedov description will be a better approximation to the truth than might otherwise be expected. This statement is of course very qualitative. A great deal more work needs to be done on cloud ionization and evaporation before it can be made more precise.

Acknowledgement: This paper was prepared while the author was visiting the Astronomy Department at Cornell University. The author thanks Professor Y. Terzian and his colleagues for their hospitality and for much enlightening discussion. In connection with this visit he also acknowledges financial assistance by the Science Research Council through grant SG/B/02390.

Comments and References

Two review articles that deal with work on supernovae up to a few years ago are by:

L. Woltjer Annual Review Astron. Astrophys. 10, 129, 1972

and

F. D. Kahn 14th International Cosmic Ray Conference, volume 11, 3566,1975

'R. A. Chevalier discusses models of the supernova explosion and the nature of their photosphere in Astrophys. J. 207, 872, 1976.

The definition of the various phases in the development of a remnant is discussed by Woltjer (op. cit.).

K. Kamper and S. v.d.Bergh give a detailed account of the structure of Cassiopaeia A in Astrophys. J. Supplement 32, 351, 1976.

The Sedov solution is described in more detail by Kahn (op.cit.). 
J. C. Raymond, D. P. Cox and B. W. Smith describe the cooling of interstellar gas in Astrophys. J. 204, 290, 1976.

The simple approximation to their calculated $\Lambda(\mathrm{T})$ comes from F. D. Kahn, Astron. Astrophys. 50, 145, 1976.

A galactic corona, with somewhat smaller density than adopted here, is described by R. A. Chevalier and R. Oergerle in Astrophys. J. $227,398,1979$.

Isothermal rather than adiabatic remnants were first discussed in detail by A. Solinger, S. Rappaport and J. Buff in Astrophys. J. $201,381,1875$. But L. L. Cowie later considered some of the restrictionson thermal conduction by electrons in Astrophys. J. 215, 226, 1977.

C. F. McKee, L. L. Cowie and J. P. Ostriker deal with the properties of inhomogeneous models of supernova remnants in Astrophys. J. 219, L23, 1978 .

The textbook "Electrodynamics of Particles and Plasmas" (Addison Wesley, 1969) by P. C. Clemmow and J. P. Dougherty contains a section (11.5) on plasma transport processes, and deals with the mirror instability in section 11.6.3.

For some primitive ideas on the ionization and evaporation of dense clouds in interstellar space see F. D. Kahn, Physica 41,172 , 1969 . 